\title{
Compatibility Conditions of the Theory of Micromorphic Elastic Solids
}

\section{A. CEMAL ERINGEN*}

This paper is a contribution in honor of T. Y. Thomas. It is an invited paper.

1. Introduction. Eringen and Suhubi [1, 2] gave a theory of microelasticity in which the local intrinsic motion of material elements is taken into account in the continuum behavior of elastic solids. This theory and its later extensions and specializations show that a large variety of physical phenomena, hitherto not encountered in the classical theories of elasticity and fluid dynamics, can be treated. For example, the effects of couple stress, distributed body couples, surface tensions, and distributed microcracks can be formulated mathematically and the underlying physical phenomena can be studied systematically. Other work related to this has appeared both in the United States and Europe. The aim of the present paper is not an assessment of existing literature, but to supply the compatibility conditions missing in these works. For a discussion of various theories, we refer the reader to [3] and [4].

2. Strain measures. According to the theory of micromorphic materials, (this terminology was introduced by Eringen [5] in his later work), the motion of a material volume element $\Delta V$ is described in terms of the "average motions" of large numbers of microvolume elements $\Delta V^{(\alpha)},(\alpha=1,2, \cdots)$ contained in $\Delta V$. The position vector of a material point in $\Delta V^{(\alpha)}$ is marked by $\mathrm{X}^{(\alpha)}=$ $\mathrm{X}+\Xi^{(\alpha)}$ where $\mathrm{X}$ is the position vector of the center of mass $P$ of $\Delta V$ and $\Xi^{(\alpha)}$ is the relative position vector of a material point of $\Delta V^{(\alpha)}$ from the center of mass $P$, Fig. 2.1. The motion of the assemblage contained in $\Delta V$ is postulated to be

$$
\mathbf{x}^{(\alpha)}=\mathbf{x}(\mathbf{X}, t)+\chi_{K}(\mathbf{X}, t) \mathbf{\Xi}_{K}^{(\alpha)},
$$

where repeated indices indicate summation over the range $(1,2,3)$. In (2.1) $\mathbf{x}(\mathrm{X}, t)$ is the spatial position $p$ of the center of mass $P$ at time $t$ and the remaining

* This work was supported by the National Aeronautics and Space Administration. 


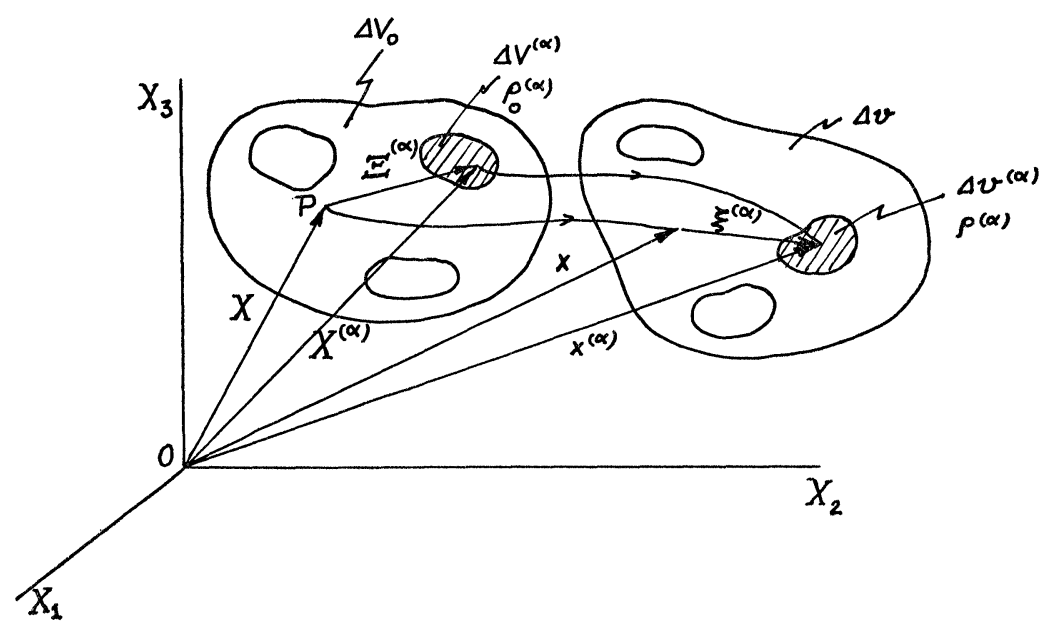

Frgure 2.1. Macromass Elements Containing Micromass Elements

terms on the right of (2.1) represent the intrinsic motion of the elements $\Delta V^{(\alpha)}$ about the center of mass. It is assumed that both "macro" and "micro"-motions

$$
\mathbf{x}=\mathbf{x}(\mathbf{X}, t), \quad \xi^{(\alpha)}=x_{K}(\mathbf{X}, t) \Xi_{K}^{(\alpha)}
$$

are continuous and possess continuous partial derivatives with respect to $X_{K}$ (the rectangular coordinates of $\mathbf{X}$ ) and time $t$ as many times as we desire. It is also assumed that the inverse motions to (2.2) exist in some neighborhood of $\mathbf{X}$ and are given by

$$
\mathbf{X}=\mathbf{X}(\mathbf{x}, t), \quad \mathbf{\Xi}^{(\alpha)}=\boldsymbol{x}_{k}(\mathbf{x}, t) \xi_{k}^{(\alpha)}
$$

except possibly at some singular surfaces, lines and points. This is secured by assuming that the jacobians not vanish,

$$
\begin{gathered}
J \equiv \operatorname{det}\left(\frac{\partial x_{k}}{\partial X_{K}}\right) \neq 0, \\
j \equiv \operatorname{det}\left(\chi_{k K}\right) \neq 0,
\end{gathered}
$$

in some neighborhood of $\mathbf{X}$ during the time interval under consideration. Here det means determinant and $\chi_{k K}$ are the components of $\chi_{K}$ referred to the spatial rectangular coordinates $x_{k}$. Similarly the components of $\boldsymbol{x}_{k}$ referred to the material rectangular coordinates $X_{K}$ are denoted by $\mathfrak{X}_{k k}$, i.e.,

$$
\chi_{K}=\chi_{k K}(\mathbf{X}, t) \dot{\mathbf{i}}_{k}, \quad \boldsymbol{x}_{k}=x_{K k}(\mathbf{x}, t) \mathbf{I}_{K},
$$

where $\dot{\mathbf{i}}_{k},(k=1,2,3)$ and $\mathbf{I}_{K},(K=1,2,3)$ are the unit base vectors of the spatial and material coordinates $x_{k}$ and $X_{K}$ respectively. Deformation gradients $\left(\mathbf{x},,_{K}, \mathbf{X},_{k}\right)$ and the microdeformations $\left(x_{K}, \boldsymbol{x}_{k}\right)$ play important roles in the theory. Among them we have the orthogonality relations 


$$
\begin{aligned}
x_{k, K} X_{K, l} & =\delta_{k l}, & X_{K, k} x_{k, L} & =\delta_{K L}, \\
\chi_{k K} x_{K l} & =\delta_{k l}, & X_{K k} \chi_{k L} & =\delta_{K L},
\end{aligned}
$$

of which (2.6) is an immediate consequence of the chain rule of differentiation and (2.7) follows from carrying one of $(2.2)_{2}$ and $(2.3)_{2}$ into the other. By considering one set of the deformation gradients and one set of the microdeformations unknown, we can solve for the other sets from the nine linear equations (2.6) and (2.7). Thus for example

$$
\begin{aligned}
X_{K, k} & =\frac{\text { cofactor } x_{k, K}}{J}=\frac{1}{2 J} e_{K L M} e_{k l m} x_{l, L} x_{m, M} \\
X_{K k} & =\frac{\text { cofactor } \chi_{k K}}{j}=\frac{1}{2 j} e_{K L M} e_{k l m} \chi_{l L} \chi_{m M}
\end{aligned}
$$

where $e_{K L M}$ and $e_{k l m}$ are the alternating symbols. For the jacobians we also have

$$
\begin{gathered}
J=\frac{1}{6} e_{K L M} e_{k l m} x_{k, K} x_{l, L} x_{m, M}, \\
j=\frac{1}{6} e_{K L M} e_{k l m} \chi_{k K} \chi_{l L} \chi_{m M} .
\end{gathered}
$$

By differentiation from (2.10) and (2.11) we also have the identities

$$
\begin{aligned}
\frac{\partial J}{\partial x_{k, K}} & =\text { cofactor of } x_{k, K}=J X_{K, k}, \\
\frac{\partial j}{\partial \chi_{k K}} & =\text { cofactor of } \chi_{k K}=j x_{K k} .
\end{aligned}
$$

Of these, (2.12) is the well-known identity of Jacobi while (2.13) is appropriate to the present theory.

The differential elements in the undeformed and the deformed bodies are calculated respectively by

$$
\begin{aligned}
& d \mathbf{X}(\alpha)=d \mathbf{X}+d \boldsymbol{\Xi}=\left(\mathbf{X}_{, k}+\boldsymbol{x}_{l, k} \xi_{l}\right) d x_{k}+\boldsymbol{x}_{k} d \xi_{k}, \\
& d \mathbf{x}(\alpha)=d \mathbf{x}+d \xi=\left(\mathbf{x}_{, K}+\chi_{L, K} \Xi_{L}\right) d X_{K}+\chi_{K} d \Xi_{K},
\end{aligned}
$$

where we drop superscripts $\alpha$ from $\Xi_{K}$ and $\xi_{k}$ and write $\mathbf{X}(\alpha)$ and $\mathbf{x}(\alpha)$ for $\mathbf{X}^{(\alpha)}$ and $\mathbf{x}^{(\alpha)}$ for simplicity. In this form $\mathbf{X}(\alpha)$ and $\mathbf{x}(\alpha)$ may be understood to denote the position vector of a material point in $\Delta V^{(\alpha)}$ and in $\Delta v^{(\alpha)}$, respectively, when the number of such microelements in $\Delta V$ and $\Delta v$ is dense.

The square of arc length $d s(\alpha)$ in the deformed body can be calculated by

$$
\begin{aligned}
& d s^{2}(\alpha)=d \mathbf{x}(\alpha) \cdot d \mathbf{x}(\alpha)=d x_{k} d x_{k}+2 d x_{k} d \xi_{k}+d \xi_{k} d \xi_{k}, \\
& d s^{2}(\alpha)=\left(C_{K L}+2 \Gamma_{K M L} \Xi_{M}+\Gamma_{R M K} \Gamma_{S N L} C_{R S}^{-1} \Xi_{M} \Xi_{N}\right) d X_{K} d X_{L} \\
& \quad+2\left(\Psi_{K L}+\Psi_{S L} \Gamma_{R M K} C_{R S}^{-1} \Xi_{M}\right) d X_{K} d \Xi_{L}+\Psi_{M L} \Psi_{N K} C_{M N}^{-1} d \Xi_{K} d \Xi_{L},
\end{aligned}
$$


where

$$
\begin{aligned}
C_{K L}(\mathbf{X}, t) & \equiv x_{k, K} x_{k, L}, \\
\Psi_{K L}(\mathbf{X}, t) & \equiv x_{k, K} \chi_{k L}, \\
\Gamma_{K L M}(\mathbf{X}, t) & \equiv x_{k, K} \chi_{k L, M}
\end{aligned}
$$

are the deformation tensors of this theory and $C_{K L}^{-1}$ is the inverse of $C_{K L}$ given by

$$
C_{K L}^{-1}=X_{K, k} X_{L, k} .
$$

Here $\mathbf{C}$ and $\mathbf{C}^{-1}$ are the Green and Piola deformation tensors of classical continuum mechanics and $\Psi \boldsymbol{\Psi}$ and $\boldsymbol{\Gamma}$ are microdeformation tensors of the theory of micromorphic materials. In this theory, therefore, to calculate length and angle changes, we need two sets of new strain measures namely $\boldsymbol{\Psi}$ and $\boldsymbol{\Gamma}$ in addition to $\mathbf{C}$ of classical continuum mechanics.

Upon introducing the displacement vector $U$ and the microdisplacement tensors $\Phi_{K L}$ by

$$
\begin{aligned}
x_{k, K} & =\left(\delta_{L K}+U_{L, K}\right) \delta_{k L}, \\
\chi_{k K} & =\left(\delta_{L K}+\Phi_{L K}\right) \delta_{k L},
\end{aligned}
$$

the deformation tensors (2.18) to (2.20) can be written as

$$
\begin{aligned}
C_{K L} & \equiv 2 E_{K L}+\delta_{K L}=\delta_{K L}+U_{K, L}+U_{L, K}+U_{M, K} U_{M, L}, \\
\Psi_{K L} & \equiv \varepsilon_{K L}+\delta_{K L}=\delta_{K L}+\Phi_{K L}+U_{L, K}+U_{M, K} \Phi_{M L}, \\
\Gamma_{K L M} & =\Phi_{K L, M}+U_{N, K} \Phi_{N L, M},
\end{aligned}
$$

where $E_{K L}$ and $\varepsilon_{K L}$, so defined, along with $\Gamma_{K L M}$ are the material strain measures of this theory.

The entire theory can be developed, equally well, in terms of the spatial deformations and strain measures (cf. [2]).

We note that there exist $6+9+27=42$ strain components which are expressed through (2.23) to (2.25) in terms of $3+9=12$ displacement components $U_{K}$ and $\Phi_{K L}$. Clearly then any arbitrary set of $\mathbf{C}, \Psi$ and $\boldsymbol{\Gamma}$ do not correspond to physically realizable displacement fields $\mathrm{U}$ and $\mathbf{\Phi}$. More precisely we may pose the question: What are the conditions for which a set of $\mathbf{C}, \mathbf{\Psi}$ and $\boldsymbol{\Gamma}$ give rise to single-valued displacement fields? This question is answered in the following section.

3. Equations of compatibility. The compatibility conditions are the restrictions that must be placed upon $\mathbf{C}, \mathbf{\Psi}$ and $\mathbf{\Gamma}$ so that single-valued continuous displacement fields $\mathbf{U}$ and $\boldsymbol{\Phi}$ exist satisfying (2.23) to (2.25). In other words they are the integrability conditions for the set of partial differential equations (2.23) to (2.25). In principle, these conditions can be obtained by elimination of the gradients of $\boldsymbol{U}$ and $\boldsymbol{\Phi}$ from these equations by successive differentiation 
and elimination. This method is, however, hopelessly awkward. As an alternative method we make use of a theorem of Riemann namely: For a symmetric tensor $a_{k t}$ to be a metric tensor for a euclidean space, it is necessary and sufficient that $a_{k l}$ be $a$ nonsingular, positive definite tensor and that the Riemann-Christoffel tensor $R_{k l m n}^{(a)}$ formed from it vanish identically. We have

$$
\begin{aligned}
R_{k l m n}^{(a)} \equiv \frac{1}{2}\left(a_{k n, l m}+a_{l m, k n}\right. & \left.-a_{k m, l n}-a_{l n, k m}\right) \\
& +a_{r s}^{-1}([l m, s][k n, r]-[l n, s][k m, r]),
\end{aligned}
$$

where $a_{k l}^{-1}$ is the inverse matrix to $a_{k l}$ and $[k l, m]$ is the Christoffel symbol of the second kind defined by

$$
[k l, m] \equiv \frac{1}{2}\left(a_{k m, l}+a_{l m, k}-a_{k l, m}\right), a_{n s}^{-1} a_{s l}=a_{n s} a_{s l}^{-1}=\delta_{n l} .
$$

To apply this theorem we need to recognize the metric tensor in the deformed and undeformed bodies. Clearly $\Psi_{K L}$ and $\Gamma_{K L M}$ cannot be used for this purpose for in general $\mathbf{\Psi}$ is not a symmetric tensor and of course $\boldsymbol{\Gamma}$ is not a second order tensor. We observe, however, that (2.17) may be expressed in another form namely

$$
d s^{2}(\alpha)=\mathcal{C}_{A B} d X_{A} d X_{B}, \quad(A, B=1,2, \cdots, 6)
$$

where we set $d \Xi_{1} \equiv d X_{4}, d \Xi_{2} \equiv d X_{5}, d \Xi_{3} \equiv d X_{6}$ and

$$
\begin{aligned}
\mathfrak{C}_{K L}= & \mathfrak{C}_{L K} \equiv C_{K L}+2 \Gamma_{(K L)}(\alpha)+\Gamma_{M K}(\alpha) \Gamma_{N L}(\alpha) C_{M N}^{-1} \\
\mathfrak{C}_{K K^{\prime}}= & \mathfrak{C}_{K^{\prime} K}=\Psi_{K K^{\prime}-3}+\Psi_{S K^{\prime}-3} \Gamma_{R K} C_{R S}^{-1} \\
\mathfrak{C}_{K^{\prime} L^{\prime}}= & \mathfrak{C}_{L^{\prime} K^{\prime}} \equiv \Psi_{M K^{\prime}-3} \Psi_{N L^{\prime}-3} C_{M N}^{-1} \\
\Gamma_{K L}(\alpha) \equiv & \Gamma_{K M L} \Xi_{M}, \\
& (K, L, M, N=1,2,3), \quad\left(K^{\prime}, L^{\prime}, M^{\prime}, N^{\prime}=4,5,6\right)
\end{aligned}
$$

where a parenthesis enclosing indices indicates the symmetric part of the quantity involved, e.g.,

$$
\Gamma_{(K L)} \equiv \frac{1}{2}\left(\Gamma_{K L}+\Gamma_{L K}\right) .
$$

In (3.3) $\mathcal{C}_{A B}$ is a nonsingular symmetric positive definite second order tensor of the euclidean six-dimensional space. Therefore, for $\mathcal{C}_{A B}$ to be a metric tensor of this space, according to a theorem of Riemann, we must have

$$
R_{A B C D}^{(\odot)}=0, \quad(A, B, C, D=1,2, \cdots, 6) .
$$

Substituting $\boldsymbol{C}$ in place of $\mathbf{a}$ in (3.1) we get

$$
\begin{aligned}
\frac{1}{2}\left(\mathfrak{C}_{A D, B C}+\mathfrak{C}_{B C, A D}\right. & \left.-\mathfrak{C}_{A C, B D}-\mathfrak{C}_{B D, A C}\right) \\
& +\mathfrak{C}^{-1 E F}([B C, F][A D, E]-[B D, F][A C, E])=0
\end{aligned}
$$


where

$$
\begin{aligned}
{[B C, F] \equiv \frac{1}{2}\left(\mathfrak{C}_{B F, C}+\mathfrak{C}_{C F, B}-\mathfrak{C}_{B C, F}\right), \quad \mathfrak{C}^{-1 A B} \mathfrak{C}_{B C}=\delta_{C}^{A}, } \\
(A, B, C, \cdots, F=1,2, \cdots, 6) .
\end{aligned}
$$

These results are valid for curvilinear coordinates.

The tensor $\mathcal{C}_{A B}$ can have a total of 21 independent components. In six dimensions the total number of nonvanishing components of $R_{A B C D}$ is 105. Thus (3.6) gives 105 equations (not all independent) for the deformation tensor $\mathfrak{C}_{A B}$. These equations are second order partial differential equations. By use of the definitions one can express these equations in terms of $\mathbf{C}, \mathbf{\Psi}$, and $\boldsymbol{\Gamma}$. The resulting expressions are too complicated to reproduce here. Instead we give the explicit forms of the compatibility conditions for the linear theory. In this case all the nonlinear terms in (3.6) are dropped reducing it to

$$
\tilde{\mathfrak{C}}_{A D, B C}+\tilde{\mathfrak{C}}_{B C, A D}-\tilde{\mathfrak{C}}_{A C, B D}-\tilde{\mathfrak{C}}_{B D, A C}=0 .
$$

For the linear theory from (3.4) we have

$$
\mathfrak{e}_{A B} \simeq \tilde{\mathfrak{e}}_{A B}
$$

where

$$
\begin{aligned}
\tilde{\mathfrak{C}}_{K L} & =\tilde{\mathfrak{C}}_{L K} \equiv \delta_{K L}+2 \widetilde{E}_{K L}+2 \widetilde{\Gamma}_{(K L)}(\alpha), \\
\tilde{\mathfrak{C}}_{K K^{\prime}} & =\tilde{\mathfrak{C}}_{K^{\prime} K} \equiv \delta_{K K^{\prime}-3}+\tilde{\mathcal{E}}_{K K^{\prime}-3}+\widetilde{\Gamma}_{K^{\prime}-3 K}(\alpha), \\
\tilde{\mathfrak{C}}_{K^{\prime} L^{\prime}} & =\tilde{\mathfrak{C}}_{L^{\prime} K^{\prime}} \equiv \delta_{K^{\prime}-3 L^{\prime}-3}-2 \widetilde{E}_{K^{\prime}-3 L^{\prime}-3}+2 \tilde{\mathcal{E}}_{\left(K^{\prime}-3 L^{\prime}-3\right)},
\end{aligned}
$$

where $\tilde{\mathbf{E}}, \tilde{\varepsilon}$ and $\tilde{\boldsymbol{\Gamma}}$ are the material strain measures of the linear theory defined by

$$
\begin{aligned}
\widetilde{E}_{K L} & \equiv \frac{1}{2}\left(U_{K, L}+U_{L, K}\right) \\
\tilde{\mathcal{E}}_{K L} & \equiv \Phi_{K L}+U_{L, K}, \\
\tilde{\Gamma}_{K L M} & \equiv \Phi_{K L, M}, \quad \tilde{\Gamma}_{K L} \equiv \widetilde{\Gamma}_{K M L} \Xi_{M} .
\end{aligned}
$$

The symbols $\delta_{K K^{\prime}-3}$ and $\delta_{K^{\prime}-3 L^{\prime}-3}$ are the usual Kronecker deltas which assume the value 1 when the indices take the same numerical values and zero otherwise, e.g.,

$$
\delta_{K K^{\prime}-3}= \begin{cases}1 & \text { when } K^{\prime}-3=K \\ 0 & \text { otherwise. }\end{cases}
$$

We note the following identities for future use

$$
\begin{aligned}
\tilde{\mathfrak{C}}_{K L, K^{\prime}} & =\tilde{\Gamma}_{K K^{\prime}-3 L}+\tilde{\Gamma}_{L K^{\prime}-3 K}, \quad \tilde{\mathfrak{C}}_{K L, K^{\prime} L^{\prime}}=0, \\
\tilde{\mathfrak{C}}_{K K^{\prime}, L^{\prime}} & =\Gamma_{K^{\prime}-3 L^{\prime}-3 K}, \quad \tilde{\mathfrak{C}}_{K K^{\prime}, L^{\prime} M^{\prime}}=0, \\
\tilde{\mathfrak{C}}_{K^{\prime} L^{\prime}, M^{\prime}} & =0 .
\end{aligned}
$$

Now decompose (3.8) into the following set of independent equations 


$$
\begin{aligned}
& \mathfrak{C}_{K L, M N}+\mathfrak{C}_{M N, K L}-\mathfrak{C}_{K N, M L}-\mathfrak{C}_{M L, K N}=0, \\
& \mathfrak{C}_{K L, M K^{\prime}}+\mathfrak{C}_{M K^{\prime}, K L}-\mathfrak{C}_{K K^{\prime}, L M}-\mathfrak{C}_{L M, K K^{\prime}}=0, \\
& \mathfrak{C}_{K L, K^{\prime} L^{\prime}}+\mathfrak{C}_{K^{\prime} L^{\prime}, K L}-\mathfrak{C}_{K L^{\prime}, L K^{\prime}}-\mathfrak{C}_{L K^{\prime}, K L^{\prime}}=0, \\
& \mathfrak{C}_{K K^{\prime}, L^{\prime} M^{\prime}}+\mathfrak{C}_{L^{\prime} M^{\prime}, K K^{\prime}}-\mathfrak{C}_{K M^{\prime}, K^{\prime} L^{\prime}}-\mathfrak{C}_{K^{\prime} L^{\prime}, K M^{\prime}}=0, \\
& \mathfrak{C}_{K^{\prime} L^{\prime}, M^{\prime} N^{\prime}}+\mathfrak{C}_{M^{\prime} N^{\prime}, K^{\prime} L^{\prime}}-\mathfrak{C}_{K^{\prime} N^{\prime}, L^{\prime} M^{\prime}}-\mathfrak{C}_{L^{\prime} M^{\prime}, K^{\prime} N^{\prime}}=0 .
\end{aligned}
$$

Upon substituting from (3.10) and using the identities (3.12) we find that the last two equations of (3.13) are satisfied identically and the first three reduce to

$$
\begin{gathered}
(3.14) \quad \widetilde{E}_{K L, M N}+\widetilde{E}_{M N, K L}-\widetilde{E}_{K N, L M}-\widetilde{E}_{L M, K N}+\frac{1}{2}\left\{\left(\widetilde{\Gamma}_{K S L}+\widetilde{\Gamma}_{L S K}\right),_{M N}\right. \\
\left.+\left(\widetilde{\Gamma}_{M S N}+\widetilde{\Gamma}_{N S M}\right),{ }_{K L}-\left(\widetilde{\Gamma}_{K S N}+\widetilde{\Gamma}_{N S K}\right),{ }_{M L}-\left(\widetilde{\Gamma}_{M S L}+\widetilde{\Gamma}_{L S M}\right),_{K N}\right\} \Xi_{S}=0, \\
(3.15) \quad \tilde{\varepsilon}_{M N, K L}-\tilde{\varepsilon}_{K N, L M}+\widetilde{\Gamma}_{K N L, M}+\widetilde{\Gamma}_{L N K, M} \\
\quad-\widetilde{\Gamma}_{L N M, K}-\widetilde{\Gamma}_{M N L, K}+\left(\widetilde{\Gamma}_{N S M, K L}-\widetilde{\Gamma}_{N S K, L M}\right) \Xi_{S}=0, \\
(3.16) \quad 2 \tilde{\varepsilon}_{(M N), K L}-2 \widetilde{E}_{M N, K L}-\widetilde{\Gamma}_{M N L, K}-\widetilde{\Gamma}_{N M K, L}=0 .
\end{gathered}
$$

Hence equations (3.14) to (3.16) are the compatibility conditions of the linear theory of micromorphic elastic solids. When $\Xi_{S}$ is not considered to be arbitrary we have eighteen independent equations instead of 105 . When $\Xi_{S}$ is considered arbitrary then the coefficients of $\Xi_{S}$ must be set equal to zero independently. This leads to

$$
\begin{aligned}
& \widetilde{E}_{K L, M N}+\widetilde{E}_{M N, K L}-\widetilde{E}_{K N, L M}-\widetilde{E}_{L M, K N}=0, \\
& \left(\widetilde{\Gamma}_{K S L}+\widetilde{\Gamma}_{L S K}\right),{ }_{M N}+\left(\widetilde{\Gamma}_{M S N}+\widetilde{\Gamma}_{N S M}\right),_{K L} \\
& -\left(\widetilde{\Gamma}_{K S N}+\tilde{\Gamma}_{N S K}\right),{ }_{M L}-\left(\tilde{\Gamma}_{M S L}+\tilde{\Gamma}_{L S M}\right),_{K N}=0, \\
& \tilde{\mathcal{E}}_{M N, K L}-\tilde{\mathcal{E}}_{K N, L M}+\tilde{\Gamma}_{K N L, M}+\tilde{\Gamma}_{L N K, M}-\tilde{\Gamma}_{L N M, K}-\tilde{\Gamma}_{M N L, K}=0 \text {, } \\
& \widetilde{\Gamma}_{N S M, K L}-\widetilde{\Gamma}_{N S K, L M}=0, \\
& 2 \tilde{\varepsilon}_{(M N), K L}-2 \widetilde{E}_{M N, K L}-\widetilde{\Gamma}_{M N L, K}-\widetilde{\Gamma}_{N M K, L}=0 .
\end{aligned}
$$

Upon using $(3.17)_{4}$ we find that $(3.17)_{2}$ is satisfied identically. The integration of $(3.17)_{4}$ gives

$$
\Gamma_{N S M, K}-\Gamma_{N S K, M}=\Omega_{N S K M},
$$

where $\Omega_{N S_{M K}}$ is independent of $\mathbf{X}$ and $\Xi$ and is subject to the restrictions

$$
\Omega_{N S(K M)}=0 .
$$

With the help of (3.18), equations $(3.17)_{3}$ and $(3.17)_{5}$ can be written as

$$
\begin{gathered}
\left(\tilde{\mathcal{E}}_{M N, K}-\tilde{\mathcal{E}}_{K N, M}+\tilde{\Gamma}_{K N M}-\tilde{\Gamma}_{M N K}\right),_{L}+\Omega_{K N M L}+\Omega_{M N L K}+\Omega_{L N M K}=0, \\
2\left[\tilde{\mathcal{E}}_{(M N), K}-\widetilde{E}_{M N, K}-\tilde{\Gamma}_{(M N) K}\right],{ }_{L}-\Omega_{M N K L}=0 .
\end{gathered}
$$


These are integrated to give

$$
\begin{aligned}
& \tilde{\mathcal{E}}_{M N, K}-\tilde{\mathcal{E}}_{K N, M}+ \tilde{\Gamma}_{K N M}-\tilde{\Gamma}_{M N K} \\
&=-\left(\Omega_{K N M L}+\Omega_{M N L K}+\Omega_{L N M K}\right) X_{L}+B_{K M N}, \\
& \tilde{\mathcal{E}}_{(M N), K}-\widetilde{E}_{M N, K}-\tilde{\Gamma}_{(M N) K}=\frac{1}{2} \Omega_{M N K L} X_{L}+D_{K M N},
\end{aligned}
$$

where $B_{K M N}$ and $D_{K M N}$ are independent of $\mathrm{X}$ and $\mathbf{\Xi}$ and are subject to

$$
B_{(K M) N}=0, \quad D_{K M N}=D_{K N M} \text {. }
$$

If the displacement field $U_{K}$ possesses continuous second order partial derivatives and the microdisplacement field $\Phi_{K L}$ possesses continuous first order partial derivatives then

$$
\Omega_{K L M N}=B_{K L M}=D_{K L M}=0,
$$

as can be seen by merely substituting $\varepsilon_{K L}, E_{K L}$ and $\Gamma_{K L M}$ from (3.11) on the left of (3.22) and (3.23).

Hence

$$
\begin{gathered}
\widetilde{E}_{K L, M N}+\widetilde{E}_{M N, K L}-\widetilde{E}_{K N, L M}-\widetilde{E}_{L M, K N}=0 \\
\tilde{\mathcal{E}}_{M N, K}-\tilde{\mathcal{E}}_{K N, M}+\widetilde{\Gamma}_{K N M}-\widetilde{\Gamma}_{M N K}=0 \\
\tilde{\mathcal{E}}_{(M N), K}-\widetilde{E}_{M N, K}-\widetilde{\Gamma}_{(M N) K}=0 \\
\tilde{\Gamma}_{K L M, N}-\widetilde{\Gamma}_{K L N, M}=0
\end{gathered}
$$

and the proof of

Theorem 1. The necessary and sufficient conditions for the integrability of the system (3.11) for a simply-connected domain is the satisfaction of the compatibility conditions (3.26) to (3.29).

When the compatibility conditions (3.26) to (3.29) are satisfied then singlevalued solutions of the system (3.11) exist for $U_{K}$ and $\Phi_{K L}$ and have the most general form

$$
\begin{gathered}
U_{K}=U_{K}^{0}+\Omega_{K L} X_{L}+B_{K}, \\
\Phi_{K L}=\Phi_{K L}^{0}+\Omega_{K L},
\end{gathered}
$$

where $\Omega_{K L}$ and $B_{K}$ are independent of $X_{K}$ and $\Xi_{K}$ and

$$
\Omega_{(K L)}=0 \text {. }
$$

Physically $\Omega_{K L} X_{L}+B_{K}$ represents a rigid body displacement field.

The compatibility conditions corresponding to the eulerian counterpart $\left(\tilde{e}_{k l}, \tilde{\epsilon}_{k l}\right.$ and $\left.\tilde{\gamma}_{k l m}\right)$ of the strain measures $\widetilde{E}_{K L}, \tilde{\varepsilon}_{K L}$ and $\tilde{\Gamma}_{K L M}$ have identical forms to (3.26) and (3.29) where capital letters are replaced by small letters and $\tilde{\boldsymbol{\Gamma}}$ is replaced by $-\tilde{\gamma}$. For the definitions of the eulerian strain measures and other related accounts the reader is referred to [1]. 
4. Compatibility conditions for micropolar elasticity. For micropolar elasticity we have, by definition

$$
\Phi_{K L}=-\Phi_{L K} \text {. }
$$

This suggests introducing an axial vector $\Phi_{K}$ such that

$$
\Phi_{K}=\frac{1}{2} \epsilon_{K L M} \Phi_{M L}, \quad \Phi_{K L}=-\epsilon_{K L M} \Phi_{M} .
$$

The linear parts of the strain measures given by (2.23) to (2.25) now read

$$
\begin{aligned}
E_{K L} & =\frac{1}{2}\left(U_{K, L}+U_{L, K}\right), \\
\mathcal{E}_{K L} & =U_{L, K}-\epsilon_{K L M} \Phi_{M}, \\
\Gamma_{K L M} & =-\epsilon_{K L N} \Phi_{N, M} .
\end{aligned}
$$

From these equations it is clear that

$$
\varepsilon_{(K L)}=E_{K L}, \quad \Gamma_{(K L) M}=0 .
$$

Using these we see that (3.28) is satisfied identically. Thus:

Theorem 2. The necessary and sufficient conditions for the integrability of the system (4.3) for a simply-connected domain is the satisfaction of the equations (3.26), (3.27) and (3.29).

These are the compatibility conditions of the linear theory of micropolar elasticity, Eringen [6]. (3.26) and (3.27) were obtained by Sandru [7].

On this singular occasion I would like to pay tribute to Professor T. Y. Thomas, a great man and a distinguished engineering scientist.

\section{REFERENCES}

[1] A. C. Eringen \& E. S. Suhubi, Nonlinear theory of simple micro-elastic solids-I, Inter. J. Eng. Sci., 2 (1964) 189-203.

[2] E. S. Suhubi \& A. C. Eringen, Nonlinear theory of simple micro-elastic solids-II, Inter. J. Eng. Sci., 2 (1964) 389-404.

[3] A. C. ERIngen, Theory of micropolar elasticity, Mathematical Fundamentals of Fracture, Vol. II, edited by H. Liebowitz, Academic Press 1967.

[4] A. C. Eringen, Mechanics of micromorphic continua, 1967 IUTAM Symposium, Freudenstadt-Stuttgart, Germany, Springer-Verlag.

[5] A. C. Eringen, Mechanics of micromorphic materials, Proc. Eleventh Inter. Congress Applied Mech., Springer-Verlag, (1966), pp. 131-138.

[6] A. C. Eringen, Linear theory of micropolar elasticity, J. Math. Mech., 15 (1966) 909-924.

[7] N. SANDRU, On some problems of the linear theory of asymmetric elasticity, Inter. J. Eng. Sci., 4 (1966) 81-96.

Princeton University 